PROCEEDINGS OF THE

AMERICAN MATHEMATICAL SOCIETY

Volume 134, Number 10, October 2006, Pages 3081-3083

S 0002-9939(06)08352-3

Article electronically published on May 4, 2006

\title{
MINIMIZING EULER CHARACTERISTICS OF SYMPLECTIC FOUR-MANIFOLDS
}

\author{
D. KOTSCHICK
}

(Communicated by Ronald A. Fintushel)

\begin{abstract}
We prove that the minimal Euler characteristic of a closed symplectic four-manifold with given fundamental group is often much larger than the minimal Euler characteristic of almost complex closed four-manifolds with the same fundamental group. In fact, the difference between the two is arbitrarily large for certain groups.
\end{abstract}

It was first proved by Dehn [2] that every finitely presentable group $\Gamma$ can be realized as the fundamental group of a closed oriented smooth four-manifold. Taking the minimum over the Euler characteristics of all such manifolds, one obtains an interesting numerical invariant $q^{D I F F}(\Gamma)$ of finitely presentable groups; see for example [4, 5, 7]. As mentioned in [7, there are geometric variants $q^{G E O}(\Gamma)$ of this definition, obtained by minimizing the Euler characteristic only over those fourmanifolds with fundamental group $\Gamma$ which carry a specified geometric structure. One trivially has

$$
q^{D I F F}(\Gamma) \leq q^{G E O}(\Gamma)
$$

for all geometric structures. Moreover, the inequality is often strict.

For a simple example of a geometric invariant, consider almost complex fourmanifolds. Every finitely presentable group is the fundamental group of an almost complex four-manifold [6], but the minimal Euler characteristic over almost complex four-manifolds is strictly larger than $q^{D I F F}(\Gamma)$ for many $\Gamma$. Nevertheless, in this case it is easy to see that the difference between the smooth and geometric invariants is universally bounded independently of $\Gamma$; compare 6 .

The purpose of this paper is to show that in the symplectic category this boundedness fails. Recall that Gompf [3] proved that every finitely presentable $\Gamma$ can be realised as the fundamental group of a closed symplectic four-manifold. Thus we can define $q^{S Y M P}(\Gamma)$ to be the minimal Euler characteristic of a closed symplectic four-manifold with fundamental group $\Gamma$. Then we have

Theorem 1. For every $c>0$ there exists a finitely presentable group $\Gamma$ satisfying

$$
q^{S Y M P}(\Gamma) \geq q^{D I F F}(\Gamma)+c .
$$

Proof. We shall use the sequence $F_{r}$ of free groups of rank $r$. It suffices to show that the difference

$$
q^{S Y M P}\left(F_{r}\right)-q^{D I F F}\left(F_{r}\right)
$$

Received by the editors May 3, 2005.

2000 Mathematics Subject Classification. Primary 57M07, 57R17, 57R57.

The author is grateful to P. Kirk for pointing out the question that is answered here. 
grows linearly with the rank $r$. We know from [7] that $q^{D I F F}\left(F_{r}\right)=-2(r-1)$, because, on the one hand, this value is the obvious lower bound $2-2 b_{1}$ for the Euler characteristic of any closed four-manifold with fundamental group $F_{r}$, and, on the other hand, this value is realized by the connected sum of $r$ copies of $S^{1} \times S^{3}$.

To estimate $q^{S Y M P}\left(F_{r}\right)$ let $X$ be a closed symplectic four-manifold with fundamental group $F_{r}$ and with minimal Euler characteristic. The minimality of the Euler characteristic implies that $X$ is symplectically minimal in the sense that it contains no symplectically embedded $(-1)$-spheres. Let us assume for the moment that the positive part $b_{2}^{+}(X)$ of the intersection form of $X$ is strictly larger than 1. Then a result of Taubes [10] implies $c_{1}^{2}(X) \geq 0$; see also [8]. We expand this inequality as follows:

$$
0 \leq c_{1}^{2}=2 \chi+3 \sigma=4-4 b_{1}+5 b_{2}^{+}-b_{2}^{-} \leq 4-4 b_{1}+5 b_{2}^{+} .
$$

This yields $b_{2}^{+} \geq \frac{4}{5}\left(b_{1}-1\right)$, and thus

$$
\chi=2-2 b_{1}+b_{2} \geq 2-2 b_{1}+b_{2}^{+} \geq-\frac{6}{5}\left(b_{1}-1\right) .
$$

Therefore we have

$$
q^{S Y M P}\left(F_{r}\right) \geq-\frac{6}{5}(r-1)
$$

showing that the difference $q^{S Y M P}\left(F_{r}\right)-q^{D I F F}\left(F_{r}\right)$ grows linearly with $r$.

It remains to remove the assumption $b_{2}^{+}(X)>1$. As $X$ is symplectic, the only other possibility is $b_{2}^{+}(X)=1$. If this happens, consider a $d$-fold covering $X_{d}$ of $X$, with $d>1$. This is symplectic with free fundamental group of rank $1+d(r-1)$. The multiplicativity of the signature and of the Euler characteristic in finite coverings then imply $b_{2}^{+}\left(X_{d}\right)=d b_{2}^{+}(X)=d>1$. We can not apply Taubes's inequality to $X_{d}$ because a priori we do not know that $X_{d}$ is symplectically minimal. Instead of proving this, we argue as follows. The minimal model $Y_{d}$ of $X_{d}$ has the same $b_{1}$ and $b_{2}^{+}$as $X_{d}$. Taubes's inequality $c_{1}^{2} \geq 0$ applied to $Y_{d}$ gives

$$
0 \leq c_{1}^{2}\left(Y_{d}\right) \leq 4-4 b_{1}\left(Y_{d}\right)+5 b_{2}^{+}\left(Y_{d}\right)=d(9-4 r) \text {. }
$$

It follows that $r \leq 2$. In the cases $r \leq 1$, inequality (1) is trivial. In the case $r=2$ it reduces to $q^{S Y M P}\left(F_{2}\right) \geq-1$, which is true because in this case $\chi(X)=$ $2-2 b_{1}(X)+b_{2}(X)=-2+b_{2}(X) \geq-1$.

This result was motivated by the recent paper of Baldridge and Kirk [1, concerned with a systematic study of $q^{S Y M P}(\Gamma)$. The lower bounds for $q^{S Y M P}(\Gamma)$ given in [1] are never better than $q^{D I F F}(\Gamma)+2$, because only the condition $b_{2}^{+} \geq 1$ and the existence of almost complex structures on symplectic manifolds are used.

It turns out that the bound (1) holds in almost complete generality.

Theorem 2. Let $\Gamma$ be a finitely presentable group. The inequality

$$
q^{S Y M P}(\Gamma) \geq-\frac{6}{5}\left(b_{1}(\Gamma)-1\right)
$$

holds for $\Gamma$ if and only if $\Gamma$ is not the fundamental group of a closed oriented surface of genus $\geq 2$.

Proof. First of all, if $\Gamma$ is the fundamental group of a closed oriented surface of genus $g \geq 2$, then it was proved in 7 ] that $q^{D I F F}(\Gamma)=4(1-g)=2\left(2-b_{1}(\Gamma)\right)$. The manifold $S^{2} \times \Sigma_{g}$ realizes the minimum and is symplectic, so that $q^{S Y M P}(\Gamma)=$ $2\left(2-b_{1}(\Gamma)\right)<\frac{6}{5}\left(1-b_{1}(\Gamma)\right)$. 
Suppose now that $\Gamma$ is not a surface group. If a symplectic manifold with fundamental group $\Gamma$ realizing the smallest possible Euler characteristic has $b_{2}^{+}>1$, then Taubes's inequality $c_{1}^{2} \geq 0$ for minimal symplectic manifolds with $b_{2}^{+}>1$ implies (2), as in the proof of Theorem 1. If the symplectic minimizer for $\Gamma$ has $b_{2}^{+}=1$, then for arbitrary $\Gamma$ we may not be able to use covering tricks as in the proof of Theorem 1. However, because $\Gamma$ is not a surface group, our manifold cannot be ruled. Therefore we can use Liu's extension 9 of Taubes's inequality to minimal non-ruled symplectic manifolds with $b_{2}^{+}=1$ to reach the same conclusion as before.

Gompf [3] asked whether a non-ruled symplectic four-manifold necessarily has non-negative Euler characteristic. This question is still open. A positive answer would of course provide a vast generalization of the results proved here. If a finitely presentable group $\Gamma$ satisfies $q^{D I F F}(\Gamma)<0$, then one knows a lot of its properties. For example, $\Gamma$ cannot embed non-trivially in itself with finite index, it is non-amenable, and has a subgroup of finite index surjecting onto $F_{2}$; see [4, 7. Thus there are many group-theoretic constraints for a negative answer to Gompf's question.

\section{REFERENCES}

1. S. Baldridge and P. Kirk, On symplectic 4-manifolds with prescribed fundamental group. Preprint arXiv:math.GT/0504345 v1 17Apr2005.

2. M. Dehn, Über unendliche diskontinuierliche Gruppen, Math. Annalen 71 (1912), 116-144. MR.1511645

3. R. E. Gompf, A new construction of symplectic manifolds, Ann. Math. 142 (1995), 527-595. MR:1356781 (96j:57025)

4. M. Gromov, Volume and bounded cohomology, Publ. Math. I.H.E.S. 56 (1982), 5-99. MR $0686042(84 \mathrm{~h}: 53053)$

5. J.-C. Hausmann and S. Weinberger, Caractéristiques d'Euler et groupes fondamentaux des variétés de dimension 4, Comment. Math. Helv. 60 (1985), 139-144. MR.0787667|(86m:57020)

6. D. Kotschick, All fundamental groups are almost complex, Bull. London Math. Soc. 24 (1992), 377-378. MR1165382 (93f:57026)

7. D. Kotschick, Four-manifold invariants of finitely presentable groups, in Topology, Geometry and Field Theory, ed. K. Fukaya et. al., World Scientific, 1994. MR.1312175 (95m:57003)

8. D. Kotschick, The Seiberg-Witten invariants of symplectic four-manifolds, Séminaire Bourbaki, 48ème année, 1995-96, no. 812, Astérisque 241 (1997), 195-220. MR1472540 (98h:57057)

9. A.-K. Liu, Some new applications of the general wall crossing formula, Math. Research Letters 3 (1996), 569-585. MR 1418572 (97k:57038)

10. C. H. Taubes, $S W \Rightarrow G r$, From the Seiberg-Witten equations to pseudo-holomorphic curves, Jour. Amer. Math. Soc. 9 (1996), 845-918. MR1362874 (97a:57033)

Mathematisches Institut, Ludwig-Maximilians-Universität München, TheresienStrasse 39, 80333 MÜNChen, Germany

E-mail address: dieter@member.ams.org 